\title{
Téoros
}

Revue de recherche en tourisme

\section{Le tourisme, un phénomène culturel. La langue, un trait culturel}

\section{Charles Dupont}

Volume 2, numéro 1, février 1983

URI : https://id.erudit.org/iderudit/1080842ar

DOI : https://doi.org/10.7202/1080842ar

Aller au sommaire du numéro

Éditeur(s)

Université du Québec à Montréal

ISSN

0712-8657 (imprimé)

1923-2705 (numérique)

Découvrir la revue

Citer ce document

Dupont, C. (1983). Le tourisme, un phénomène culturel. La langue, un trait culturel. Téoros, 2(1), 27-29. https://doi.org/10.7202/1080842ar d'utilisation que vous pouvez consulter en ligne.

https://apropos.erudit.org/fr/usagers/politique-dutilisation/ 


\section{Le tourisme, un phénomène culturel La langue, un trait culturel

\author{
par Charles Dupont
}

\section{N.D.L.R}

Lors du congrès Langue et Société au Qué bec qui s'est tenu du 11 au 13 novembre 1982 a Québec, un atelier portait sur le tourisme et le français. L'atelier, structuré autour de quatre questions precises, visait a êtudier l'incidence négative ou positive du français dans l'image touristique du Québec. Nous reproduisons ici l'expose de Charles Dupont qui répond a ces quatre questions.

\section{1) L'image du Québec francais par} les médias étatsuniens et canadiens anglophones influence-t-elle la venue des touristes?

A ma connaissance aucune donnée n'existe sur cette question, et l'étude de Mme Bourgeois ${ }^{11 !}$, qui donne une bonne idee de l'acceptation du fait français de la part des touristes anglophones au Québec, n'aborde pas non plus cet aspect. Par exemple, dans le questionnaire on ne demandait pas aux gens silis avaient déjâ lu des articles sur la politique linguistique du Quebec et, dans l'affirmative, si cela les avait fait hésiter à venir chez nous. Et comment comptabiliser ceux qui ont pu être influencés par ces articles?

L'argument de l'image negative du Québec français semble plutôt fonde sur des rumeurs aux contours imprécis, et place dans le contexte international il a l'air mème un peu exagerere. Faut-il ne plus visiter Madrid, Paris, ou Londres parce que d'innocents touristes ont été tués lors d'atten. tats terroristes? Faut-il fuir les grandes villes étatsuniennes parce que souvent il faut limiter ses déplacements pedestres après le coucher du soleil?

\section{2) Les Québécois sont-ils privés} d'informations touristiques importantes parce qu'on doit leur fournir en français? S'il s'agit des dépliants visant à interesser les Quebecois francophones à visiter le Québec je ne vois pas comment; s'il s'agit d'intéresser les Québécois anglophones et allophones, peut-étre y a-t-ill lá un problème. Mais des statistiques ${ }^{27}$ indiquent que les Québécois anglophones sont, parmi les Canadiens, ceux qui voyagent le moins au Canada $139,1 \%$ en 1978 alors que dans toutes les régions ce taux dépasse $57 \%$ l et ceux qui visitent le moins leur province de résidence $114,1 \%$ en 1978 alors qu'ailleurs le taux du tourisme intraprovincial depasse $41 \%)$.
Enfin s'il s'agit du tourisme international des Québecois, il suffit de suivre l'évolution du deficit touristique du Quebec pour compren dre que les Québécois ne manquent pas d'informations pour se rendre à l'étranger.

Les deux autres questions, vu de l'angle de mes préoccupations, semblent davantage toucher l'essentiel de la refflexion que le Congrès cherche à stimuler.

3) La francisation du Québec crée-t-elle un attrait touristique nouveau pour les Canadiens et en particulier pour les Etatsuniens?

Ma première réaction à cette question serait de dire: pourquoi le devrait-elle? La promotion du catalan à Barcelone et dans toute la Catalogne fait-elle la manchette des journaux dans le monde, et en fait-on un "attrait touristique"? Le finnois, qui n'avait pas encore droit de cité á l'université d'Helsinki il $y$ a moins de cinquante ans, est aujourd hui une langue en mesure de rivali. ser en fonction et en efficacité avec les gran des langues de civilisation, et cela sans qu'il $n$ 'ait eu à renier ses origines nous affirme-t. on ${ }^{13}$. En fait-on pour cela un attrait touristi. que? Pas a ce que je sache, mais ce fait temoigne du dynamisme d'une culture et de la confiance en soi d'un peuple dont le niveau de vie est aujourd hui parmi les plus elevés au monde.

Comme facteur isolé, la francisation du Qué bec ne constitue pas a mon avis un attrait touristique pour les Canadiens et les Etatsuniens, bien que cela puisse être plausible si on ajoutait à cette liste les francophones du monde entier (Francais, Belges, Libanais, Marocains, s soit environ 90 millions de personnes.

Toutefois je miempresse d'ajouter que la francité du Québec n'est pas non plus un "désattrait touristique". Je vois plutót cette francite comme un elément ou un trait d'un ensemble plus grand que iaborderai plus loin.

Avant je veux analyser brievement le tou risme réceptif au Québec sur le plan des motivations de nos visiteurs, et essaver de soupeser l'incidence du fait français sur leur choix.

Suivant une conception courante du tou. risme. les touristes se divisent en trois grands groupes: al les touristes d'affaires: b) les touristes d'agrément; c) les touristes de congrès.

a) Les touristes d'affaires se rendent là où il y a des transactions commerciales possiblès, là où l'activité économique est fébrile. La langue $n$ 'a aucune incidence sur leur choix. A ce type de tourisme on peut associer le tourisme industriel, c.-ad. la visite de réailsations techniques. Si quelqu'un veut voir le plus grand barrage du monde c'est ici qu'il va le trouver, et la langue que l'on parle n'importe guere.

b) Les touristes d'agrément ont des motivations fort diverses, parfois inextricables et même insondables si on se fie aux ettudes publiées sur le sujet. Quelle est l'importance du facteur francité dans le cas des deplacements suivants:

- pour visiter parents et amis: faible ou nulle:

- pour chasser ou pour pécher: la quantité du gibier ou de poissons compte süre. ment plus:

- pour les activités de plein air: le touriste est súrement plus soucieux de trouver des rivières et des lacs non pollués par les pluies acides, des campings propres. etc. que du fait que l'on parle français;

- le tourisme itinerant incidence sürement plus forte, mais une etude de I'OTC révele que le vacancier étatsunien recher. che d'abord l'amabilite du visité et un service aimable, ce que je crois qu'il peut trouver au Quebec:

- pour uñ pelerinage: la foi transcende les langues

- pour des vacances en famille: la langue peut avoir une incidence négative ou positive lpar ex. permettre aux enfants de baragouiner les quelques mots de français appris à l'ecolel, mais la possibilité de trouver des activités pour les entants sera un facteur encore plus déterminant:

- pour la gastronomie: tout le monde sait qu'on ne parle pas la bouche pleine et que la terminologie française dans ce domaine est largement connue.

Bref à mon avis le facteur francité ne doit pas non plus être exagére comme attrait ou desattrait touristique en ce qui concerne les voyages d'agrément. 
c) Les touristes de congrès

C'est habituellement de ce côté qu'on entend le plus de "grogne esur les désavantages de la francisation du Québec. Pourtant I'article 14 du Règlement relatif à la langue du commerce et des affaires stipule que l'article 58 de la loi 101 ne stapplique pas "lors d'un congrès, d'une foire, d'une exposition ou d'un colloque destine /... / a un public specialise...." is

C'est donc en vain qu'on cherche à faire de la francisation le bouc emissaire d'une sta gnation apparente du tourisme de congrès, stagnation d'ailleurs bien minime aux yeux de Jean Labonté, président de la Société du Palais des Congrès de Montréal, car malgré les difficultés économiques actuelles le secteur congrès maintient une croissance d'environ $10 \%$ en dollars courants ${ }^{6}$.

Donc, quand on veut parler de l'incidence du français sur le tourisme au Québec, il faut bien se garder de prendre l'arbre pour la forêt.

4) Si la francisation ou le visage francais ne constitue ni un attrait ni un désattrait touristique, peut-on quand mềme l'utiliser à l'avantage du tourisme et de l'industrie touristique

Ideux choses qu'il ne faut pas toujours confrondrel?

Je répondrais sans hésiter oui. Mais comment?

Si josais dire en n'en parlant pas, ce ne serait pas tout à fait juste, mais je dirais pré sentons le comme un simple trait de culture. C'est ce que je voulais exprimer plus tốt quand jaai souligné que notre francité n'était. selon moi, qu'un element d'un ensemble plus important, c'est-à-dire notre culture, ce que nous sommes.

A mon avis le mot culture est indissociable du phénomène touristique car comme le déclarait le secrétaire general de l'Organisa tion mondiale du tourisme (OMT) lors de la journee mondiale du tourisme le 27 septem. bre dernier

"on ne peut pas penser bendificer d'un deplacement, quelle que soit la motrivation, si d'un coté comme de l'autre, on ne fait pas preuve d"un préalable essentiel à savoir que la vie sur notre planete ne releve pas d'une froide normalisation inanimee deja établie, mais que sa richesse, sa beauté et sa forme exaltante d'évolution résident dans sa diversite, dans son foisonnement d'images aussi attravantes les unes que les autres ${ }^{\text {Tinat. }}$.

Cette prise de position cadre bien d'une part avec la déclaration de 1979 du Comite de promotion du tourisme culturel selon laquelle "le tourisme doit devenir un agent culturel positif, c'est-adire, un agent susceptible de mettre en valeur et de respecter les façons de vivre des Québecois, leur patrimoine, leur environnement et leur langue ${ }^{(8) *}$, et d'autre part avec les articles XIII et XIV du Projet de Code du touriste en voie d'élaboration par l'OMT, articles qui portent sur les devoirs du touriste et qui stipulent entre autres que celui-ci doit:

- "respecter le patrimoine culturel et natu. rel des visites:

- faire preuve de la plus grande compré. hension a l'égard des usages, coutumes, croyances et comportements des communautés d'accueil:

- éviter d'accentuer les différences eco. nomiques, sociales et cu/turelles exis. tant entre eux et la population locale;

- s'ouvrir a la culture de la communauté d'accueil, partie integrante du patrimoine culturel de l'humanite"

L'élément culture occupe donc une place primordiale en tourisme, et la langue est probablement un des premiers traits d'une culture qu' un visiteur doit accepter. En principe aucun touriste canadien anglais ou etatsunien ne viendra au Québec seulement parce qu'on y parle le français (pas plus qu'on va aux Etats. Unis uniquement pour entendre parler l'anglais), mais il peut venir parce qu'on y présente un trait distinctif, soit la vie en trançais dans le confort nordaméricain, et parce qu'un touriste c'est dans la très grande majorité des cas une personne qui cherche un minimum de dépaysement. Et je dirais meme qu'il faut le lui procurer si on desire qu'il revienne.

Sur un plan plus pratique, nous pourrions aussi profiter de notre situation linguistique en commercialisant les sejours d'immersion linguistique dans nos ecoles de langue à l'exemple de l'Angleterre, de la France, de l'Espagne, etc. Ce créneau reste un vaste champ à exploiter, et si des études etaient réalisées, peut-titre serions-nous en mesure de dire que la langue aussi rapporte!

Cela étant dit, la langue n'est pas une chose que l'on place dans les musées, mais une chose qui vit, qui soulève les passions let je ne pense pas uniquement aux Québế. coisl, qui doit servir la communication à tous les niveaux entre les membres d'un groupe linguistique et, surtout, quil reflète la viva cité intellectuelle d"un peuple, et donc d'une culture comme les Etatsuniens en font la preuve chaque jour.

Par contre, un peuple incapable d'exprimer le présent et de prefigurer l'avenir dans sa langue fait la preuve de son manque de dynamisme et de confiance en soi, et je regrette mais cela jette souvent la même lumière sur les professionnels des divers secteurs économiques.

Quelle est la réalité linguistique dans l'indus trie touristique?

Gilles Vigneault disait l'autre jour a la radio "qu'il en est des langues comme des peu. ples, elles ne som belles que dans la mesure ou elles sont authentiques, autonomes et libres ${ }^{* 2}$.
Mais quand nous vovons la qualite de laf. fichage où fleurissent les expressions comme: heure heureuse (pour le cina à sept ou l'heure de l'apéritif), spécial du jour (pour plat du jourl, camper ou maison motorisee (pour autocaravane), plan europeen (sur les permis du gouvernement du Québec dans les chambres d'hötels ou de motelsil.

- quand on en est rendu à employer le mot "hospitalite" pour parler d'une industrie lévidemment en anglais on dit hospita. lity industryl.

- quand I'ITHQ et le MITC acceptent dans la prèsentation d'un projet étudiant très valable une expression comme "audio tour" alors qu'il aurait fallu dire tout simplement sonoguide.

- ou encore quand on entend un politique responsable du tourisme parler de "saison touristique epaule" (on aura reconnu le shoulder season) au lieu de saison intermediaire.

je me dis que le français d'ici n'est plus authentique, autonome, libre et qu'il a mème perdu un peu de ses racines, contrairement au finnois dont j'évoquais l'exemple tout à I'heure. Par rapport à d'autres secteurs et compte tenu de ses pretentions fort justifiees à mon avis, car après tout le tourisme ce r'est pas le commerce de la ferraille, l'industrie touristique n'a pas fait l'effort qu'on aurait eté en droit d'attendre d'elle en matière de francisation en général et de qua lité de la langue en particulier.

Notre sensibilisation à cette réalité dans le domaine du tourisme, l2e industrie au Qué becl est-elle récente? Oui car ce thème a pour la première tois eté aborde près de nous a l'occasion de la Vlle Biennale de la langue française qui s'est tenue en 1977 à P'Université de Moncton. A cette occasion Louis Martin Tard declarait:

"Si on demandart a la langue françarse comment elle va dans le domaine du tourisme, elle repondrait: Assez mal merci. Affige d'hypertrophie verbale et surtout d'anglicité aigüe, certe pauvre langue touristique, toute affaiblie qu'elle est, semble se laisser aller." 110

Cette situation est-elle normale? Dans notre contexte on serait tenté de répondre oui, car pourquoi la situation devrait-elle ètre meilleure dans le secteur touristique que dans celui de l'automobile ou tout autre. Mais si on accepte lidee que le tourisme est une excellente activité où le visité et le visiteur entrent en contact dans des rapports égaux, c.al.d. quion depasse la simple notion de commerce. l'attitude de nos protessionnels est paradoxale car bien qúils oeuvrent à l'intérieur de ce secteur dont l'essence est d'inviter les vacanciers au dépaysement et â la decouverte d'autres peuples, coutumes, moeurs, etc., ils n'en anglicisent et n'en appauvrissent pas moins notre vocabulaire du tourisme sous prétexte de l'internationaliser. 
Leur question est toujours la méme: Ie fran cais a-t-il les ressources pour exprimer les réalités du tourisme moderne? Poser la question c'est y repondre. Le probleme il faut le chercher du cóté des parlants fran. çais qui sont tellement obnubiles par la lan gue anglaise qu'ils ignorent les ressources de leur langue, voient des trous terminologiques lá oủ il n'y en a pas, et offrent une résistance suicidaire aux termes bièn créés en français. Parfois on a l'impression qu'il est aussi difficile de remplacer un terme anglais par un terme français, que de réus. sir un soufflé, ou qu'il s'agit d'un crime de lèse-majesté.

II a été prouvé qu"il est possible de traduire par des mots simples des expressions comme fast food/restauration- minute, fifth wheel/semi-caravane ou motor home/autocaravane. Pout ne citer quiun autre exemple, celui de voyagiste qui est la traduction de tour operator, il est étonnant de voir la résistance des protessionnels, alors que dejaz en 1978 I'Office de tourisme du Canada l'adoptait.

Pour tout dire, et cela jette aussi un éclairage sur l'élement culture abordé plus tôt, l'avenir du francais touristique nest pas assure. car les protessionnels n'y accordent pas d'importance et peu d'enseignants des écoles publiques et privées de tourisme semblent se soucier d'inculquer aux étudiants les appellations justes des notions. et cela meme s'il est reconnu que l'apprentissage de la terminologie d'une discipline est une bonne facon d'en saisir toutes les facettes:

Bref, selon moi la culture est un élément essentiel pour attirer des touristes, la langue est un élément important de la culture d'un peuple, le français peut être chez nous une langue vivante, contemporaine et dynamique, et le dynamisme attire les touristes. Enfin si les Jamaicains ont raison d'afficher bien en vue des touristes artivant dans leur pays "Jamaica is not a beach, it is a country", le Québec, tout en traitant cha: que visiteur comme un invité attendu, n'a pas à s'excuser auprès des touristes ou a leur cacher que le francais fait partie du Qué bec, comme le rocher Percé et les paysa. ges de Charlevoix, et il a mème intérèt à êtudier toutes les façons d'en tirer profit.

"C'est une erreur de sous-estimer le pouvoir des mots, et des idees qu'incarnent les mots". (Jimmy Carter, Discours aux étudiants de I'Universite Notre-Dame $\left.{ }^{1 / 1}\right)$ f

\section{Reférences}

11) BOURGEOS. Manon, GIRA RD, Henes. Renctions des tou risies face a ut tranciation do latfichace of de Le pubto

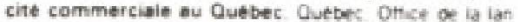
que francase $198 \mathrm{E}$ \% 90

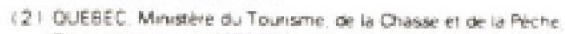


tion Traveller Canadan in 1978

(3) DESNEIGES, Geonges Finlande, Pass 5eul 1574. Petite planese. D. 16

(4) OFFICE DE TOUAISME DU CANADA. Noweties strmege: de communcation pour les. E. U.". In Canade Teuriame. wal 2 no 7 salembre 1982 . $p$ i

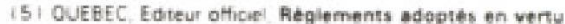
de la Charte de la ingue trancaise mars $19 \mathrm{~B}$

16. Le tourisme, force de happe de nome pcomome. in Höne: Lerio Restaurstion, vol. 56 no 8 seplember $198 \mathrm{~g}$. po 10.12

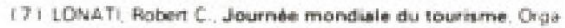

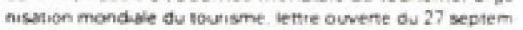
wet 198 ?

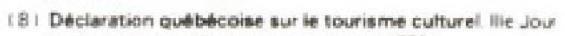

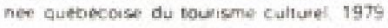

I9. ORGARISATION MONDALE DU TOUARSME Phoiet de

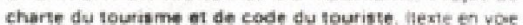
de elaborsionil Madnd

(nO) FEDERATION DU FRANCAIS UNIVERSEL Langue fran caise et identite cuhuralie - Moncton (1977). Dakar Les Nouvelies editons afrcaines. 1979 of 326

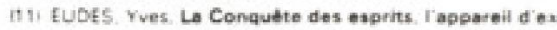
portation cuturalle americain. Psis, Maspeco 1962 p. $2 t$

\section{TÉOROS}

\section{Ne manquez pas les prochains numéros!}

\section{Année 1983}

Les deux prochains numeros paraitront

- à la fin juin 1983 (vol. 2, no. 2): Analyse des attractions touristiques du Quebec.

- à la fin octobre 1983 (vol. 2, no. 3): Urbanisme, amenagement et tourisme.

\section{Avez-vous de vieilles photos sur le Québec?}

Dans le cadre du prochain numero portant sur les attractions touristiques au Québec, nous souhaitons présenter un bilan très documenté sur tout ce qu'on a pu dire, ecrire, diffuser à propos du Québec et de ses résidents pour attirer les visiteurs. Nous voulons appuyer les témoignages ecrits par des photos d'époque lpouvant dater du début du sieciel sur les attraits et les attractions touristiques du Québec.

Si wous possédez de telles photos, nous vous invitons da nous les faire parvenif. Quelques-unes de ces photos seront selectionnees et paraitront dans le prochain numéro avec les mentions d'usage.

Veuillez nous indiquer si vous désirez que nous vous retournions ces photos ou si vous nous les laissez pour fins de publications uiterieures.

Merci de votre collaboration

\author{
Adresse pour l'envoi des \\ photos: \\ Téoros \\ Cahiers de recherche en tourisme \\ Université du Québec à Montréal \\ C.P. 8888, Succursale "A" \\ Montréal H3C 3P8
}

